\title{
Cervical tuberculous lymphadenopathy: changing clinical pattern and concepts in management
}

\author{
B C Jha, A Dass, N M Nagarkar, R Gupta, S Singhal
}

\begin{abstract}
Tuberculosis is one of the biggest health challenges the world is facing. In this study the clinical pattern of patients with cervical lymphadenitis, who presented to the ear, nose, and throat outpatient department of the Government Medical College Hospital, Chandigarh, India between June 1997 and May 1998 is recorded. Tuberculosis accounted for 60 out of 94 cases of cervical lymph node enlargement. The commonest age group affected was 11-20 years. Constitutional symptoms were not present in most of the patients. Multiple matted nodes were seen in 23 patients but a single discrete node was seen in 18 patients. Upper deep jugular nodes were the most commonly affected lymph nodes. Discharging sinus and abscess formation were uncommon. Fine needle aspiration cytology yielded a positive diagnosis in 52 out of 56 patients. Chest lesions on radiography were evident in $16 \%$ of the patients. Mantoux test was positive and was more than $15 \mathrm{~mm}$ in most of the patients. This study shows that the classical picture of "scrofula" is no longer seen nowadays and can probably be explained by the earlier presentation of the disease.
\end{abstract}

All the patients were treated with short course daily chemotherapy for six months. Surgery was not required in the majority of patients except in four cases where excision biopsy was performed. Patients with abscess formation were managed with wide bore needle aspiration only. With a minimum six month period of follow up, no patient was found to have a recurrence of local or systemic disease.

This study emphasises the role of fine needle aspiration cytology in diagnosis and confirms the efficacy of six months short course chemotherapy.

(Postgrad Med f 2001;77:185-187)

Keywords: cervical lymphadenitis; tuberculosis; antitubercular chemotherapy

Tuberculosis continues to be the biggest health problem in developing countries with enormous social and economic implications. Even in the developed countries, where the disease was controlled to a large extent, it is again posing a new health challenge. This is due to the migration of people from developing areas with a high prevalence of tuberculosis and the increasing high incidence of HIV infection in these countries. This has resulted in a worldwide resurgence of tuberculosis. During the past decade the clinical pattern and presentation of tuberculosis has changed dramatically. Much of the traditional learning about this disease is no longer true and tuberculosis has become a new entity. ${ }^{1}$

Tuberculous lymphadenitis is the commonest form of extrapulmonary tuberculosis. Cervical lymph nodes are the most common lymph nodes affected by this disease-classically termed as "scrofula". We studied the recent clinical pattern of the disease because we come across many patients with cervical tuberculous lymphadenopathy in our outpatient department. Although literature is available on various aspects of this disease, not many recent studies have been done on the clinical pattern of the disease. We treated our patients with short course chemotherapy with very few patients requiring surgery. Our experience with this treatment modality is presented here.

\section{Patients and methods}

The present study involved 56 patients with tuberculous cervical lymphadenopathy, out of the total 94 patients with cervical lymphadenopathy, who attended the ear, nose, and throat outpatient department of the Government Medical College Hospital, Chandigarh, India from June 1997 to May 1998. This institution caters to a population of 900000 .

After a detailed history and clinical examination, fine needle aspiration cytology of involved nodes was performed in all these patients. Biopsy of the lymph nodes was performed on the two occasions when fine needle aspiration cytology was either negative or doubtful and clinical suspicion was high for tuberculosis. Routine tests including erythrocyte sedimentation rate (ESR), Mantoux test, chest radiography, and sputum examination for acid-fast bacilli were also done in all the patients. HIV screening was not performed in any of the patients as there was no clinical suspicion regarding AIDS.

After confirmation of diagnosis all patients were treated with short term intensive chemotherapy for six months. In the first two months isoniazid, rifampicin, pyrazinamide, and ethambutol or streptomycin was given followed by isoniazid and rifampicin for the next four months.

All the patients were followed up at monthly intervals for at least six months and progress was assessed by clinical examination as well as three monthly ESR estimation.

\section{Results}

Tuberculosis was the commonest cause of cervical lymphadenopathy accounting for 60 out of 94 cases. Out of these 60 patients, 56 cases 
were included in the present study. Four patients were lost to follow up and were excluded from the study. Other causes of cervical lymphadenopathy were metastatic (18 patients), non-specific inflammation (13 patients), and lymphoma (three patients).

The age of these patients ranged from 9 months to 62 years with a mean age of 23.7 years. The commonest age group affected by the disease in this study was $11-20$ years (23 patients) followed by 21-30 years (20 patients). There were 24 males and 32 females (M:F ratio was $1: 1.3$ ). Thirty five patients $(62.5 \%)$ belonged to the lower socioeconomic group.

The time interval between onset of symptoms and time of presentation varied from 15 days to 36 months (mean three months). The main symptoms at the time of presentation are summarised in table 1 .

Cervical lymph nodes alone were involved in 49 patients. Cervical and axillary lymph nodes were involved in five patients and cervical and inguinal lymph nodes were enlarged in two patients.

Examination of cervical lymph nodes revealed multiple matted nodes in 23 cases, a single discrete node was present in 18 cases, and multiple discrete nodes were seen in nine cases. Cervical abscesses and discharging sinuses were seen in three patients each.

The most common group of lymph nodes involved was the upper deep jugular (including jugulodigastric), followed by jugulo-omohyoid and submandibular. Abscess and sinus formation were most common in supraclavicular group of lymph node - that is, out of five there were two abscesses and one sinus. Lymph nodes were involved on one side in 38 patients and bilateral involvement was present in 18 patients.

Fine needle aspiration cytology revealed a positive diagnosis in 48 cases in first aspiration $(85.7 \%)$ and was positive on second aspiration in four cases. In four cases lymph node excision and biopsy was needed to confirm the clinical diagnosis.

The ESR was raised in all but four cases and it was more than $30 \mathrm{~mm}$ in the first hour in most of the cases. Mantoux test was positive in 53 patients. It was more than $20 \mathrm{~mm}$ in 15 patients, between $15-20 \mathrm{~mm}$ in 32 patients, and between $10-15 \mathrm{~mm}$ in six patients. In three patients either reaction was negative or less than $5 \mathrm{~mm}$ in transverse diameter. Associated chest lesions on radiography were evident only in nine cases $(16 \%)$ : pleural effusion in four cases, upper zone infiltrate in three cases, and primary complex in two cases (both children).

Table 1 Symptomatology in patients with cervical tuberculous lymphadenopathy

\begin{tabular}{lc}
\hline Symptoms & No (\%) of patients \\
\hline Neck swelling & $53(94.6)$ \\
Malaise & $10(17.8)$ \\
Weight loss & $8(14.3)$ \\
Fever & $6(10.7)$ \\
Cough & $6(10.7)$ \\
Discharging sinus & $3(5.3)$ \\
Haemoptysis & $1(1.8)$ \\
\hline
\end{tabular}

Sputum for acid-fast bacilli was positive in two cases only $(3.6 \%)$.

The size of the lymph node was reduced to less than $5 \mathrm{~mm}$ within three months of starting full dose chemotherapy in more than $40 \%$ of cases. After six months $82 \%$ of them had reduction of size to less than $5 \mathrm{~mm}$. After nine months, only $6 \%$ of patients had a lymph node of more than $5 \mathrm{~mm}$, but they had a normal ESR and were free of any constitutional symptoms. Surgery other than excision biopsy was not needed in any patient. Multiple aspirations using a wide bore needle were needed only in patients with abscesses. Two of these patients developed abscesses after starting chemotherapy. The abscesses and sinuses healed within four months. To date none of the patients has shown any recurrence of local or systemic disease with minimum follow up of six months after completion of chemotherapy.

\section{Discussion}

Tuberculosis, "Captain of all these men of death", as referred to by John Bunyan in the 18 th century is still the biggest health challenge of the world. It is known that $1.5 \%$ of India's population is affected with tuberculosis. ${ }^{2}$ There are no recent studies regarding the background prevalence of tuberculosis in Chandigarh.

Peripheral lymph node tuberculosis is the most common form of extrapulmonary tuberculosis. Cervical tuberculous lymphadenopathy or "scrofula", which used to be treated in medieval times in England by the "King's touch" and issuance of a "gold coin", is still the most common cause of persistent cervical lymph node enlargement in the developing countries.

We encountered 60 patients with cervical tuberculous lymphadenopathy out of 94 patients who had cervical lymph node enlargement $(63.8 \%)$. In a study by Dandapat et al although this disease accounted for $43 \%$ of such cases, this was still the commonest diagnosis for lymph node enlargement. ${ }^{3}$

Although traditionally taught as one of the causes of generalised lymphadenopathy, the involvement of the axillary or inguinal group of lymph nodes was not common in this study. Similar views were suggested by other investigators. ${ }^{3-5}$

The commonest age group affected in our study was $11-20$ years followed by $21-30$ years. This was also noted in the study by Subrahmanyam. ${ }^{6}$ But no age is a bar as we have seen cases in infants as well as in those aged more than 60 years. In the USA and the UK, the highest incidence of tuberculous lymphadenitis occurs between 25 and 50 years of age. ${ }^{78}$ The ratio of males to females in this study was $1: 1.3$, which is similar to that found by Dandapat et al (1:1.2) and Subrahmanyam $(1: 1.3) .^{36}$ Dandapat et al thought that this may be because females are more conscious of their appearance and in the male dominated society they have a low nutritional status. ${ }^{3}$

In our study most of the patients did not have constitutional symptoms. The most common systemic symptoms of malaise and weight loss were found in only $18 \%$ and $14 \%$ of 
patients respectively. Even fewer patients had cough, fever, or haemoptysis. Although in a series of 100 patients, Patel and Mehta observed weight loss in $77 \%$ and fever in $73 \%$ cases. $^{9}$ Similarly Dandapat et al also noted weight loss in $85 \%$ and fever in $40 \%$ of their patients. ${ }^{3}$ So in this respect our observation differs significantly from those of others.

According to Desa the three features which help to establish the diagnosis of tuberculous lymphadenitis are multiplicity, matting, and caseation. $^{10}$

In our study although patients having multiple matted nodes form the largest group $(47 \%)$, the number of patients having single discrete node was quite significant (32\%). The number of patients having cervical abscess or sinus was quite low ( $5 \%$ each). This finding is contrary to those of Cheung et al: $22 \%$ of their patients at the time of presentation had an abscess or a discharging sinus. ${ }^{11}$

The most common group of lymph nodes affected in this study was that of the upper deep jugular and jugulodigastric node. This is similar to the finding of Dandapat et al. ${ }^{3}$ In a study from Khartoum by Kheiry and Ahmed the most affected nodes were in the posterior triangle. ${ }^{12}$ Associated lung involvement as detected by chest radiography was seen in only $16 \%$ of cases in this study. This figure is quite low compared with the $40 \%-50 \%$ described in the textbook, Clinical Tuberculosis. ${ }^{13}$

Fine needle aspiration cytology is a well established diagnostic tool in assessment of cervical masses. In the developing countries where tuberculous infection is common and other granulomatous diseases are rare, the presence of granulomatous features on fine needle aspiration cytology is highly suggestive of tuberculosis. ${ }^{14}$ In our study we found it a very useful diagnostic tool to identify the patients of tuberculous lymphadenopathy positively. It obviates the need for excisional biospy in most of the patients. Similar views were expressed by others. ${ }^{15-17}$ The Mantoux test and ESR were useful adjuncts in helping to make the diagnosis.

Before the advent of chemotherapy, surgical excision of all the lymph nodes was the mainstay of treatment for tuberculous lymphadenopathy. ${ }^{18}$ In 1950 s, when chemotherapy for tuberculosis was just introduced, excision of all involved lymph nodes followed by antituberculous chemotherapy for 12-24 months was found more effective treatment. ${ }^{19}$ Cambell et al confirmed the success of a daily six months' regimen for tuberculous lymphadenopathy. ${ }^{20}$ In this study, we successfully treated all the patients with short course chemotherapy for six months. Surgery was rarely required. Our study confirms the effectiveness of a six months' regimen of chemotherapy alone for tuberculous lymphadenitis. This is similar to the experience of Patel and Mehta and Yuen et $a l .{ }^{9}{ }^{21}$

The authors would like to thank the Director Principal and the Medical Superintendent of the Government Medical College Hospital, Chandigarh for granting permission to use the hospital records. The authors acknowledge the secretarial help of Ms Rupinder Kaur in preparing the manuscript.

\section{Learning points}

- Tuberculosis is still the commonest cause of cervical lymph node enlargement in developing countries.

- Disease is relatively more common in younger patients (10-30 years).

- A single discrete node can be the presentation in many of these patients.

- Constitutional symptoms were absent in most of the patients. Chest lesions were also not common in these patients.

- Discharging sinus and abscess formation is now relatively rare in cervical tuberculous lymphadenitis.

- Fine needle aspiration cytology is a rapid, cheap, simple, and effective method of diagnosis for cervical lymphadenopathy.

- Most of the patients can be managed with a six month daily chemotherapy regimen. Surgery is rarely needed.

1 Canova CR, Kuhn M, Reinhart WH. Problems in the diagnosis and therapy of lymph node tuberculosis in HIVnegative patients. Schweiz Med Wochenschr (Fournal Suisse de Medicine) 1995;125:2511-7.

2 Mukherjee AK. Tuberculosis control programme in India: progress and prospects. Indian fournal of Tuberculosis 1995;42:75.

3 Dandapat MC, Mishra BM, Dash SP, et al. Peripheral lymph node tuberculosis: a review of 80 cases. $\mathrm{Br} \mathcal{F}$ Surg 1990;77:911-2.

4 Kent DC. Tuberculous lymphadenitis: not a localised disease process. Am f Med Sci 1967;254:866-73.

5 Challapalli M, Varnado SC, Cunningham DG. Tuberculous inguinal lymphadenitis. Pediatr Infect Dis f 1995;14:723-4. 6 Subrahmanyam M. Role of surgery and chemotherapy for 6 Subrahmanyam $M$. Role of surgery and chemotherapy for
peripheral lymph node tuberculosis. Br F Surg 1993;80: 1547-8.

7 Alvarez S, MeCabe WR. Extrapulmonary tuberculosis revisited: a review of experience at Boston city and other hospitals. Medicine 1984;63:25-53.

8 Monie RDH, Hunter AM, Rocchiccioli KMS, et al. Management of extrapulmonary tuberculosis (excluding miliary and meningeal) in South and West Wales (1976-8). BMf 1982;285:415-8.

9 Patel RV, Mehta RT. Short term chemotherapy in tuberculous lymphadenitis. Indian fournal Surgery 1987;49:336-41.

10 Desa AE. Tuberculosis of lymph glands. In: Rao KN, ed. Text book of tuberculosis. New Delhi: Vikas Publishing House, 1981: 476-81.

11 Cheung WL, Siu KF, Ng A. Tuberculous cervical abscess comparing the result of total excision against simple incision and drainage. Br $\mathcal{F}$ Surg 1988;75:563-4.

12 Kheiry J, Ahmed ME. Cervical lymphadenopathy in Khartoum. Fournal of Tropical Medicine \& Hygiene 1992;95: 416-9.

13 Humphries MJ, Lam WK, Teoh R. Non-respiratory tuberculosis. In: Davies PDO, ed. Clinical tuberculosis. London: Chapman \& Hall Medical, 1994: 93-125.

14 Lau SK, Wei WI, Hsu C, et al. Efficacy of fine needle aspiration cytology in the diagnosis of tuberculous cervical lymphadenopathy. $\mathcal{F}$ Laryngol Otol 1990;104:24-7.

15 Shaha A, Webber C, Marti J. Fine needle aspiration cytology in diagnosis of cervical lymphadenopathy. Am F Surg 1986; 152:420-3.

16 Lau SK, Wei WI, Kwan S, et al. Combined use of fine-needle aspiration cytologic examination and tuberculin skin test in the diagnosis of cervical tuberculous lymphadenitis: a prospective study. Arch Otolaryngol Head lymphadenitis: a prospective
Neck Surg 1991;117:87-90.

17 Rajwanshi A, Bhambhani S, Das DK. Fine needle aspiration cytology in diagnosis of tuberculosis. Diagn Cytopathol 1987;3:13-6.

18 Dowd CN. The surgical treatment of tubercular cervical lymph nodes. Ann Surg 1905;42:49-74

19 Byrd RB, Bopp KR, Gracey DR, et al. The role of surgery in tuberculous lymphadenitis in adults. Am Rev Respir Dis 1971;103:816-20.

20 Cambell IA, Ormerod LP, Friend JAR, et al. Six month versus nine months chemotherapy for tuberculosis of lymph nodes: final result. Respir Med 1993;87:621-3.

21 Yuen APW, Wong SHW, Tam CM, et al. Prospective randomized study of thrice weekly six-month and ninemonth chemotherapy for cervical tuberculous lymphadenopathy. Otolaryngol Head Neck Surg 1997;116:189-92. 